\title{
Jameel's Dimensional Stressed Default Probability Models are Indeed IFRS 9 Complaint Models
}

\author{
Jamilu Auwalu Adamu ${ }^{1}$ \\ ${ }^{1} 118$ National Mathematical Centre, Abuja, Nigeria \\ Correspondence: JamiluAuwaluAdamu, 118 National Mathematical Centre, Abuja, Nigeria. Tel: 234-8038-679- \\ 094. E-mail: whitehorseconsult@yahoo.com
}

Received: August 31, 2018; Accepted: October 9, 2018; Published: October 21, 2018

\begin{abstract}
Default Probabilities can be used to Analysis Firm Creditworthiness, calculate Expected Credit Losses, Economic and Regulatory Capitals for Banking Institutions and Ranking such as FICO for consumers or Bond Ratings from S \& P, Fitch or Moodys for Corporations and Governments. Banks and other Institutions are heavily investing towards Building, Developing, Improving and or Purchasing Credit Risk Models that would enhance their capabilities to handle, predict and quantify Credit Risk challenges which will subsequently help them to accurately calculate and assign sufficient Economic and Regulatory Capitals. It is believe that the existing Default Probability Models failed to accurately predict the unforeseen level of borrower defaults and resulting losses they had to recognize. The IASB in July, 2014 issued the final version of IFRS 9 Measurement of Financial Instruments beginning on or after $1^{\text {st }}$ January, 2018 with early adoption permitted. It replaces IAS 39 Financial Instruments: Recognition and Measurement. IFRS 9 require adjustments to the use of Probability of Default (PD), Exposure at Default and Loss Given Default (LGD) estimates. Probability of Default (PD) plays very important role when Calculating Expected Credit Losses under IFRS 9. Jamilu (2015) enhanced LOGIT and PROBIT Default Probability Models with the aid of one-dimensional forward-looking information $\{f(x)\}$ satisfies Jameel's Criterion and positive average of economic forecasts of future macroeconomic scenarios $\left\{\mu_{A}>\right.$ 0 , infinitesimal and $\sigma_{A} \geq 1$ \}. This paper further enhance LOGIT and PROBIT Default Probability Models using Two and Three Dimensional Forward-Looking Information(s) $\left\{f_{1}(x), f_{2}(x)\right\}$ and $\left\{f_{1}(x), f_{2}(x), f_{3}(x)\right\}$ respectively satisfies Jameel's Criterion with LOG-LOGISTIC $(3 \mathrm{P}) \equiv f_{1}(x)$, CAUCHY $\equiv f_{2}(x)$ and BURR(4P) $\equiv f_{3}(x)$ and positive average of economic forecasts of future macroeconomic scenarios $\left\{\mu_{A}\right.$ and $\left.\sigma_{A}\right\}$. The paper tested the performances of only proposed Default Probability Models of TYPES 1 in each class using Twenty One (21) working days (from 12/1/ 2014 to 12/30/2014). The results were fascinatingly interesting, impressive, viable, reliable, sophisticated, and complaint with IFRS 9 since they incorporated forwardlooking information(s) and Economic forecasts of the future macroeconomic scenarios thereby minimizing the differences between MODELS DEFAULT PROBABILITIES and REAL LIFE DEFAULT PROBABILITIES.
\end{abstract}

Keywords: Logit, Probit, Probability, Forward-Looking Information, Macroeconomic scenarios, Jameel's Criterion

JEL Classification: A1, C1, C5, C6, E6

\section{Introduction}

In July, 2014 IASB issued the final version of IFRS 9 Measurement of Financial Instruments beginning on or after $1^{\text {st }}$ January, 2018 with early adoption permitted. It replaces IAS 39 Financial Instruments: Recognition and Measurement. The major target of accounting standards is to provide financial information that stake-holders would find useful when making decisions. The most challenging aspects required by IFRS 9 are the treatment on incorporation of forward-looking information and economic forecasts of future macroeconomic scenarios into the existing Default Probability Models. The IFRS 9 accounting rules regarding Measurement of Financial Instruments will NORROW the wide gaps between Models Default Probabilitiesand Real Life Default Probabilities.

Probability of Default can be used to Analysis Firm Creditworthiness, Credit-adjusted Valuation, Economic Capital Calculations, Cash Flow and net income Analyses of firm's obligations, Ranking Firms with the same agency Credit Rating based on estimated default probabilities, Capital Provisioning, Expected Credit Losses, Economic and Regulatory Capital for Banking Institution. Barnaby Black, ShirishChinchalkar, Juan M. Licari 
(2016) argued that Regulatory Stress Testing requires that the models should demonstrate sensitivity to macroeconomic conditions.

In response to the credit crisis of 2007-2008, the banking sector adopted international financial regulations to lessen their exposure to default risk. The Basel Committee on Banking Supervision's (BCBS) Goal is to improve the existing banking sector's strategies, processes and ability to deal with FINANCIAL STRESS effectively. Under IFRS 9 Credit Risk Modelling, IFRS 9 reason was that “... the Credit Risk at Origination is included in the Pricing of Financial Asset but any increase in Credit Risk is NOT".

It is believe that the existing Default Probability Models failed to accurately predict the unforeseen level of borrower defaults and resulting losses they had to recognize. Financial Institutions are devoting serious amount of time, energy and resources towards building, developing and purchasing CREDIT RISK MODELS that may improve their abilities to predict and quantify CREDIT RISKS they faces. These credit risk models can adequately improve their abilities to sufficiently calculate Economic and Regulatory Capital reserves. These efforts have been recognized and promoted by Bank Regulators and their Macro prudential Policies.

To address Bankers and Regulators late complain that "If only we had seen this coming or had been better prepared...” Jamilu (2015) used Jameel's Contractional-Expansional Stressed Methods and Jameel's Criterion to CAME UP with Advanced Stressed Models capable of capturing IFRS 9 INCREASE IN CREDIT RISK that is NOT included at the origination in the PRICING of Financial Assets, Derivatives and Expected Credit Losses (ECLs) components (PD, EAD, LGD) as argued under IFRS 9 Credit Risk Modelling. The objectives of this paper is to further enhance LOGIT and PROBIT Default Probability Models using Two and Three Dimensional ForwardLooking Information(s) $\left\{f_{1}(x), f_{2}(x)\right\}$ and $\left\{f_{1}(x), f_{2}(x), f_{3}(x)\right\}$ respectively satisfies Jameel's Criterion with LOG-LOGISTIC (3P) $\equiv f_{1}(x)$, CAUCHY $\equiv f_{2}(x)$ and BURR(4P) $\equiv f_{3}(x)$ and positive average of economic forecasts of future macroeconomic scenarios $\left\{\mu_{A}>0\right.$, Infinitesimal and $\left.\sigma_{A} \geq 1\right\}$.

\section{Materials and Methods}

\subsection{Materials}

2.1.1 International Financial Reporting Standards 9 (Ifrs 9)
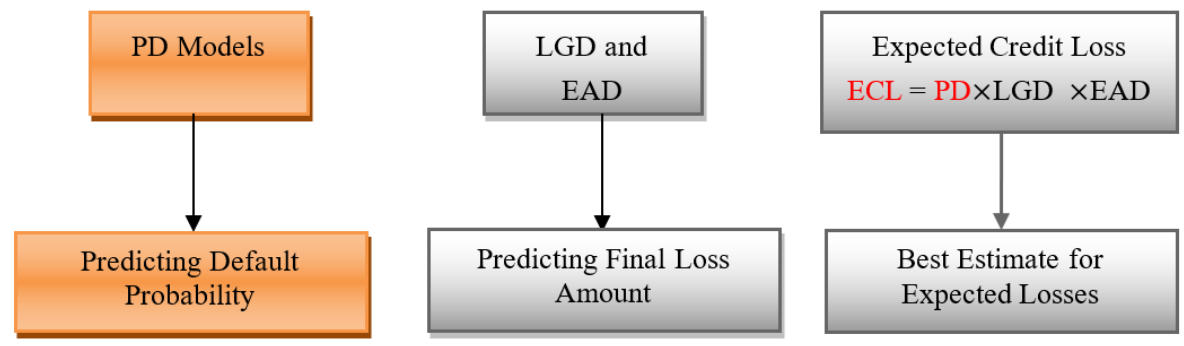

IFRS 9 require adjustments to the use of Probability of Default (PD), Exposure at Default and Loss Given Default (LGD) estimates. As from the above figure, Probability of Default (PD) plays very important role when Calculating Expected Credit Losses under IFRS 9.

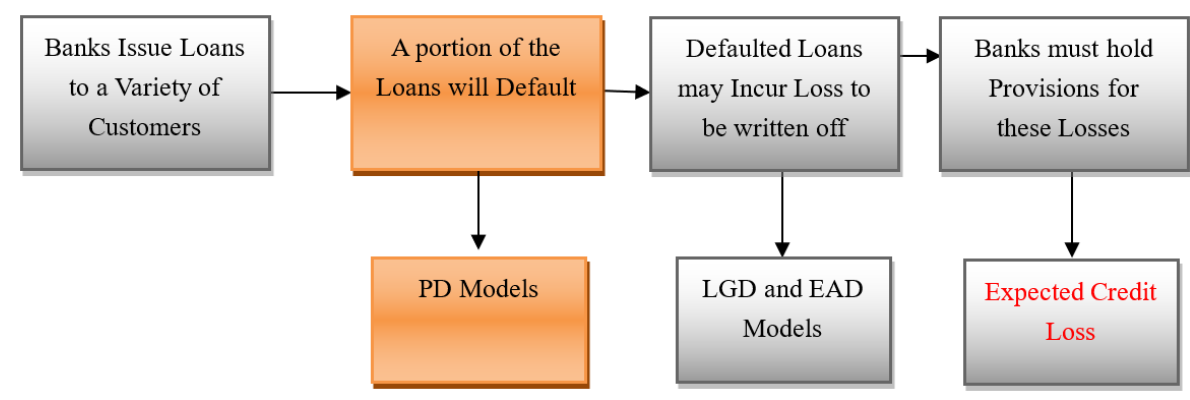

The modelling approach for the key risk parameters will be affected by the incorporation of forward - looking, credible and robust economic scenarios into ACCOUNTING MODELS. Banks faces number of challenges in meeting their designed level of IFRS 9 requirements for instance SOPHASTICATED MODELLING EXPECTATIONS, CORRECT MODELS, PEOPLES and SKILLS. 


\subsubsection{Probability of Default (Pd) or Default Probability}

This can be defined as a term describing the likelihood of a Default over a particular time horizon. It provides an estimate of the likelihood that a borrower will be unable to meet its debt obligations. PD is used in a different Credit Analyses and Risk Management Frameworks.

\subsubsection{Jameel'S Criterion}

Under this criterion, we run the goodness of fits test such that:

i. We accept if the Average of the ranks of Kolmogorov Smirnor, Anderson Darling and Chi-squared is less than or equal to Three (3)

ii. We must choose the Probability Distribution follows by the data ITSELF regardless of its Rankings

iii. If there is tie, we include both the Probability Distributions in the selection

iv. At least Two (2) Probability Distributions must be included in the selection

v. We select the most occur Probability Distribution as the qualify candidate in each case of test of goodness of fit.

vi. Criterion Enhancement Axiom:Thode (2012) intensively discussed about the Best Goodness of Fit Tests such as Kolmogorov Smirnov (KS) Test, Anderson-Darling Test, Jarque and Bera (JB) Test, Shapiro Wilk (SW) Test, Cramer-Von Mises Test, Pearson ( $\chi^{2}$ Godness of Fit) Test, Lilliefors Corrected K-S Test, D’AgostinoSkewness Test, Anscombe-Glynn Kurtosis Test, D’Agostino-Pearson Omnibus Test. Let $\left\{T_{1}, T_{2}, \ldots, T_{n}\right\}$ be the set of such Best Goodness of Fit Tests, $\left\{x_{1}, x_{2}, \ldots, x_{n}\right\}$ be their RANKS respectively then the generality of (i) can be expressed (or enhanced) if $\frac{\left(x_{1}+x_{2}+\ldots+x_{n}\right)}{n} \leq a$, where $0<a \leq n, n \in N$ or equivalently, $x_{1}+x_{2}+\ldots+x_{n} \leq a n$.

vii. Last Unit Axiom: let $W_{J B}(t)$ be such that it satisfied axioms (i) to (iv). Let $\left\{r_{1}, r_{2}, \ldots, r_{n}\right\}$ be the ranks of fitness test of $W_{J B}(t)$ obtained from the tests $\left\{T_{1}, T_{2}, \ldots, T_{n}\right\}$ respectively then if $\forall i \in\{1,2, \ldots, n\}, r_{i}=1$ regardless of the Time Series, Company and so on. Consequently, if for all fitness test runs, turn out to be the same $W_{J B}(t)$ then the PREDICTED PRICE PATH will finitely coincides many times with the

REAL PRICE PATH of the stock under consideration.

\subsection{Methods}

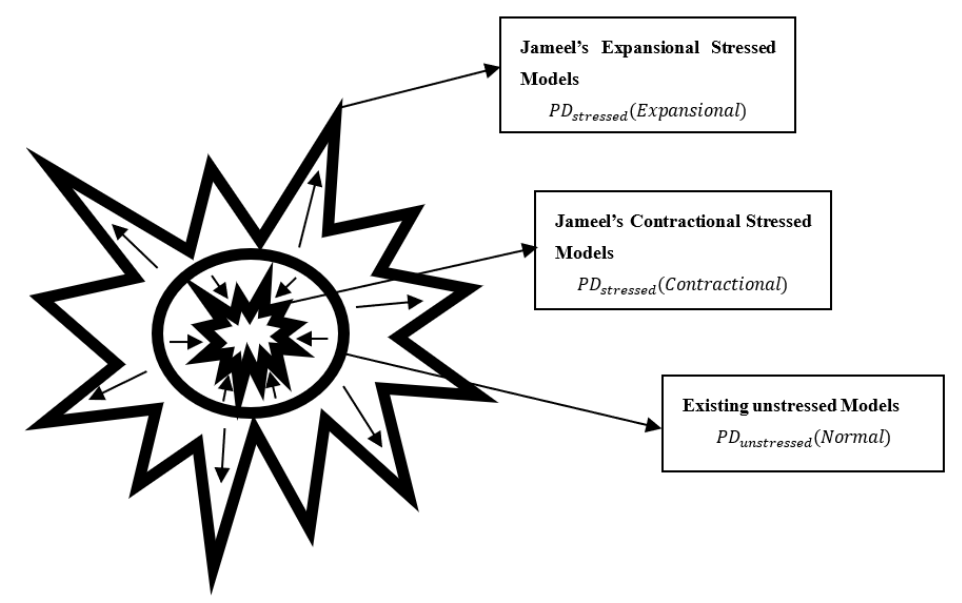

Figure 1. Jameel'sContractional-Expansional Stressed Methods

From figure 1, the basic Idea was to initially use Jameel's Contractional-Expansional Stress Methods to incorporate Low-Probability, High-Impact into the Default Probability Models: LOGIT and PROBIT Models using 
Geometric Volatility $\sigma_{A}$ and Geometric Return $\mu_{A}$ of the Arithmetic Means of the underlying Asset Return plus Returns of the explained (independent) variables as well as Jameel's Criterion based Best fitted fat-tailed Probability Distribution of the underlying Asset Return $f\left(x, \mu_{\text {company }}, \sigma_{\text {company }}, \xi\right)$ as worked out below:

- SHRINKING the NORMAL Probability of Default $P D_{\text {unstressed }}$ (Normal) to CONTRACTIONAL Probability of Default $P D_{\text {stressed }}$ (Contractional) using economic forecasts of future macroeconomic scenarios of Geometric Volatility $\sigma_{A} \geq 1$ and Only positive Geometric Return $\mu_{A}>0$, infinitesimal of the Arithmetic Means of the underlying Asset Return plus Returns of the future macroeconomicparameters as well as Jameel's Criterion Best fitted fat-tailed forward-looking information $\left\{f_{1}(x), f_{2}(x)\right\}$ for Two Dimensional and $\left\{f_{1}(x), f_{2}(x), f_{3}(x)\right\}$ for Three Dimensional of the underlying Asset Return, where $f_{1}(x), f_{2}(x)$ and $f_{3}(x)$ are $1^{\text {st }}, 2^{\text {nd }}$ and $3^{\text {rd }}$ Distributions Ranking according to Jameel's Criterion.

- BLOWING the NORMAL Probability of Default $P D_{\text {unstressed }}$ (Normal) to EXPANSIONAL Probability of Default $P D_{\text {stressed }}$ (Expansional) using economic forecasts of future macroeconomic scenarios of Geometric Volatility $\sigma_{A} \geq 1$ and Only positive Geometric Return $\mu_{A}>0$, infinitesimal of the Arithmetic Means of the underlying Asset Return plus Returns of the future macroeconomic parameters as well as Jameel's Criterion Best fitted fat-tailed forward-looking information $\left\{f_{1}(x), f_{2}(x)\right\}$ for Two Dimensional and $\left\{f_{1}(x), f_{2}(x), f_{3}(x)\right\}$ for Three Dimensional of the underlying Asset Return, where $f_{1}(x), f_{2}(x)$ and $f_{3}(x)$ are $1^{\text {st }}, 2^{\text {nd }}$ and $3^{\text {rd }}$ Distributions Ranking according to Jameel's Criterion.

2.2.1 Logit Default Probability Model

$$
P D=\frac{1}{1+\exp \left(\sum_{i=0}^{K} \beta_{i} X_{i}\right)}
$$

PD is the probability of default. $X=\left(X_{1}, X_{2}, \ldots, X_{k}\right)$ is a vector of explanatory variables (Macro-economic Indicators).

2.2.2 Probit Default Probability Model

$$
P D=\Phi\left(\beta_{0}+\sum_{j=1}^{J} \beta_{j} X_{j}\right)
$$

2.2.3 Propose Two-Dimensional Stressed Logit Default Probability Models

TYPE 1:

TYPE 2:

$$
P D_{\text {Stressed }}=\frac{1}{1+\exp \mu_{A}\left(\sum_{i=0}^{k} \beta_{i} X_{i}\right) \pm \sigma_{A} f_{1}(x) \pm \sigma_{A} f_{2}(x)}
$$

$$
P D_{\text {Stressed }}=\frac{1}{1+\exp \mu_{A}\left(\sum_{i=0}^{k} \beta_{i} X_{i}\right) \pm f_{1}(x) \pm \sigma_{A} f_{2}(x)}
$$

TYPE 3:

TYPE 4:

$$
P D_{\text {Stressed }}=\frac{1}{1+\exp \mu_{A}\left(\sum_{i=0}^{k} \beta_{i} X_{i}\right) \pm \sigma_{A} f_{1}(x) \pm f_{2}(x)}
$$

TYPE 5:

$$
P D_{\text {Stressed }}=\frac{1}{1+\exp \mu_{A}\left(\sum_{i=0}^{k} \beta_{i} X_{i}\right) \pm f_{1}(x) \pm f_{2}(x)}
$$

$$
P D_{\text {Stressed }}=\frac{1}{1+\exp \left(\sum_{i=0}^{k} \beta_{i} X_{i}\right) \pm \sigma_{A} f_{1}(x) \pm \sigma_{A} f_{2}(x)}
$$

2.2.4 Propose Three-Dimensional Stressed Logit Default Probability Models

TYPE 1:

$$
P D_{\text {Stressed }}=\frac{1}{1+\exp \mu_{A}\left(\sum_{i=0}^{k} \beta_{i} X_{i}\right) \pm \sigma_{A} f_{1}(x) \pm \sigma_{A} f_{2}(x) \pm \sigma_{A} f_{3}(x)}
$$


TYPE 2:

TYPE 3:

$$
P D_{\text {Stressed }}=\frac{1}{1+\exp \mu_{A}\left(\sum_{i=0}^{k} \beta_{i} X_{i}\right) \pm f_{1}(x) \pm \sigma_{A} f_{2}(x) \pm \sigma_{A} f_{3}(x)}
$$

TYPE 4:

$$
P D_{\text {Stressed }}=\frac{1}{1+\exp \mu_{A}\left(\sum_{i=0}^{k} \beta_{i} X_{i}\right) \pm \sigma_{A} f_{1}(x) \pm f_{2}(x) \pm \sigma_{A} f_{3}(x)}
$$

$$
P D_{\text {Stressed }}=\frac{1}{1+\exp \mu_{A}\left(\sum_{i=0}^{k} \beta_{i} X_{i}\right) \pm \sigma_{A} f_{1}(x) \pm \sigma_{A} f_{2}(x) \pm f_{3}(x)}
$$

TYPE 5:

$$
P D_{\text {Stressed }}=\frac{1}{1+\exp \mu_{A}\left(\sum_{i=0}^{k} \beta_{i} X_{i}\right) \pm f_{1}(x) \pm f_{2}(x) \pm \sigma_{A} f_{3}(x)}
$$

TYPE 6:

TYPE 7:

$$
P D_{\text {Stressed }}=\frac{1}{1+\exp \mu_{A}\left(\sum_{i=0}^{k} \beta_{i} X_{i}\right) \pm \sigma_{A} f_{1}(x) \pm f_{2}(x) \pm f_{3}(x)}
$$

TYPE 8:

$$
P D_{\text {Stressed }}=\frac{1}{1+\exp \mu_{A}\left(\sum_{i=0}^{k} \beta_{i} X_{i}\right) \pm f_{1}(x) \pm \sigma_{A} f_{2}(x) \pm f_{3}(x)}
$$

$$
P D_{\text {Stressed }}=\frac{1}{1+\exp \mu_{A}\left(\sum_{i=0}^{k} \beta_{i} X_{i}\right) \pm f_{1}(x) \pm f_{2}(x) \pm f_{3}(x)}
$$

TYPE 9:

$$
P D_{\text {Stressed }}=\frac{1}{1+\exp \left(\sum_{i=0}^{k} \beta_{i} X_{i}\right) \pm \sigma_{A} f_{1}(x) \pm \sigma_{A} f_{2}(x) \pm \sigma_{A} f_{3}(x)}
$$

2.2.5 Propose Two-Dimensional Stressed Probit Default Probability Models

TYPE 1:

$$
P D_{\text {Stressed }}=\phi\left[\mu_{A}\left(\beta_{0}+\sum_{j=1}^{J} \beta_{j} X_{j}\right)\right] \pm \sigma_{A} f_{1}(x) \pm \sigma_{A} f_{2}(x)
$$

TYPE 2:

$$
P D_{\text {Stressed }}=\phi\left[\mu_{A}\left(\beta_{0}+\sum_{j=1}^{J} \beta_{j} X_{j}\right)\right] \pm f_{1}(x) \pm \sigma_{A} f_{2}(x)
$$

TYPE 3:

$$
P D_{\text {Stressed }}=\phi\left[\mu_{A}\left(\beta_{0}+\sum_{j=1}^{J} \beta_{j} X_{j}\right)\right] \pm \sigma_{A} f_{1}(x) \pm f_{2}(x)
$$

\section{TYPE 4:}

$$
P D_{\text {Stressed }}=\phi\left[\mu_{A}\left(\beta_{0}+\sum_{j=1}^{J} \beta_{j} X_{j}\right)\right] \pm f_{1}(x) \pm f_{2}(x)
$$


TYPE 5:

$$
P D_{\text {Stressed }}=\phi\left[\left(\beta_{0}+\sum_{j=1}^{J} \beta_{j} X_{j}\right)\right] \pm \sigma_{A} f_{1}(x) \pm \sigma_{A} f_{2}(x)
$$

2.5.6 Propose Three-Dimensional Stressed Probit Default Probability Models

TYPE 1:

$$
P D_{\text {Stressed }}=\phi\left[\mu_{A}\left(\beta_{0}+\sum_{j=1}^{J} \beta_{j} X_{j}\right)\right] \pm \sigma_{A} f_{1}(x) \pm \sigma_{A} f_{2}(x) \pm \sigma_{A} f_{3}(x)
$$

TYPE 2:

$$
P D_{\text {Stressed }}=\phi\left[\mu_{A}\left(\beta_{0}+\sum_{j=1}^{J} \beta_{j} X_{j}\right)\right] \pm f_{1}(x) \pm \sigma_{A} f_{2}(x) \pm \sigma_{A} f_{3}(x)
$$

TYPE 3:

$$
P D_{\text {Stressed }}=\phi\left[\mu_{A}\left(\beta_{0}+\sum_{j=1}^{J} \beta_{j} X_{j}\right)\right] \pm \sigma_{A} f_{1}(x) \pm f_{2}(x) \pm \sigma_{A} f_{3}(x)
$$

TYPE 4:

$$
P D_{\text {Stressed }}=\phi\left[\mu_{A}\left(\beta_{0}+\sum_{j=1}^{J} \beta_{j} X_{j}\right)\right] \pm \sigma_{A} f_{1}(x) \pm \sigma_{A} f_{2}(x) \pm f_{3}(x)
$$

TYPE 5:

$$
P D_{\text {Stressed }}=\phi\left[\mu_{A}\left(\beta_{0}+\sum_{j=1}^{J} \beta_{j} X_{j}\right)\right] \pm f_{1}(x) \pm f_{2}(x) \pm \sigma_{A} f_{3}(x)
$$

TYPE 6:

$$
P D_{\text {Stressed }}=\phi\left[\mu_{A}\left(\beta_{0}+\sum_{j=1}^{J} \beta_{j} X_{j}\right)\right] \pm \sigma_{A} f_{1}(x) \pm f_{2}(x) \pm f_{3}(x)
$$

TYPE 7:

$$
P D_{\text {Stressed }}=\phi\left[\mu_{A}\left(\beta_{0}+\sum_{j=1}^{J} \beta_{j} X_{j}\right)\right] \pm f_{1}(x) \pm \sigma_{A} f_{2}(x) \pm f_{3}(x)
$$

TYPE 8:

$$
P D_{\text {Stressed }}=\phi\left[\mu_{A}\left(\beta_{0}+\sum_{j=1}^{J} \beta_{j} X_{j}\right)\right] \pm f_{1}(x) \pm f_{2}(x) \pm f_{3}(x)
$$

TYPE 9:

$$
P D_{\text {Stressed }}=\phi\left[\left(\beta_{0}+\sum_{j=1}^{J} \beta_{j} X_{j}\right)\right] \pm \sigma_{A} f_{1}(x) \pm \sigma_{A} f_{2}(x) \pm \sigma_{A} f_{3}(x)
$$


2.2.7 Propose Jameel'S N-Dimensional Stressed Default Probability Theorem

Let $\left\{W_{J B 1}(x), W_{J B 2}(x), W_{J B 3}(x), \ldots, W_{J B n}(x)\right\}, x$ is the Asset's Returns be a set of Non-Normal Fat-tailed Probability Distributions satisfies Jameel's Criterion with RANKING $1^{s t}, 2^{\text {nd }}, 3^{\text {rd }}, \ldots$, nth respectively. Let $\sigma_{A}$ be a Geometric Volatility of only positive Arithmetic Means of the Underlying Asset Return and Returns of the future economic forecasts of macroeconomic parameters and $\mu_{A}>0$, Infinitesimal be a Geometric Means of only positive Arithmetic Means of the Underlying Asset Return and Returns of the future economic forecasts of macroeconomic parameters such that:

$$
\begin{gathered}
L_{1}(x):=\exp \mu_{A}\left(\sum_{i=0}^{k} \beta_{i} x_{i}\right) \pm \sigma_{A} W_{J B 1}(x) \pm \sigma_{A} W_{J B 2}(x) \\
L_{2}(x):=\exp \mu_{A}\left(\sum_{i=0}^{k} \beta_{i} x_{i}\right) \pm \sigma_{A} W_{J B 1}(x) \pm \sigma_{A} W_{J B 2}(x) \pm \sigma_{A} W_{J B 3}(x) \\
L_{3}(x):=\exp \mu_{A}\left(\sum_{i=0}^{k} \beta_{i} x_{i}\right) \pm \sigma_{A} W_{J B 1}(x) \pm \sigma_{A} W_{J B 2}(x) \pm \sigma_{A} W_{J B(n-10)}(x) \\
L_{4}(x):=\exp \mu_{A}\left(\sum_{i=0}^{k} \beta_{i} x_{i}\right) \pm \sigma_{A} W_{J B 1}(x) \pm \sigma_{A} W_{J B 2}(x) \pm \sigma_{A} W_{J B(n-5)}(x) \pm \sigma_{A} W_{J B(n-4)}(x) \\
L_{n}(t):=\exp \mu_{A}\left(\sum_{i=0}^{k} \beta_{i} x_{i}\right) \pm \sigma_{A} W_{J B 1}(x) \pm \sigma_{A} W_{J B 2}(x) \pm \cdots \pm \sigma_{A} W_{J B n}(x)
\end{gathered}
$$

Such that

$$
P D_{\text {Stressed }}=\frac{1}{1+L_{i}(x)}, i=1,2, \ldots, n
$$

And that

$$
\begin{gathered}
L_{1}(x):=\phi\left[\mu_{A}\left(\beta_{0}+\sum_{j=1}^{J} \beta_{j} x_{j}\right)\right] \pm \sigma_{A} W_{J B 1}(x) \pm \sigma_{A} W_{J B 2}(x) \\
L_{2}(x):=\phi\left[\mu_{A}\left(\beta_{0}+\sum_{j=1}^{J} \beta_{j} x_{j}\right)\right] \pm \sigma_{A} W_{J B 1}(x) \pm \sigma_{A} W_{J B 2}(x) \pm \sigma_{A} W_{J B 3}(x) \\
L_{3}(x):=\phi\left[\mu_{A}\left(\beta_{0}+\sum_{j=1}^{J} \beta_{j} x_{j}\right)\right] \pm \sigma_{A} W_{J B 1}(x) \pm \sigma_{A} W_{J B 2}(x) \pm \sigma_{A} W_{J B(n-10)}(x) \\
L_{4}(x):=\phi\left[\mu_{A}\left(\beta_{0}+\sum_{j=1}^{J} \beta_{j} x_{j}\right)\right] \pm \sigma_{A} W_{J B 1}(x) \pm \sigma_{A} W_{J B 2}(x) \pm \sigma_{A} W_{J B(n-5)}(x) \pm \sigma_{A} W_{J B(n-4)}(x) \\
L_{n}(t):=\phi\left[\mu_{A}\left(\beta_{0}+\sum_{j=1}^{J} \beta_{j} x_{j}\right)\right] \pm \sigma_{A} W_{J B 1}(x) \pm \sigma_{A} W_{J B 2}(x) \pm \cdots \pm \sigma_{A} W_{J B n}(x)
\end{gathered}
$$

Such that

$$
P D_{\text {Stressed }}=L_{i}(x), i=1,2, \ldots, n
$$

Then we generated a set $\left\{L_{1}(x), L_{2}(x), L_{3}(x), \ldots, L_{n}(x)\right\}$ of ARBITRARY COMBINATIONS EXCLUDING FIRST FEW TERMS OF DIFFERENT DIMENSIONS. Then $\exists L_{i}(x), i=1,2, \ldots, n$ such that: 
(i) $P D_{\text {Stressed }}=\frac{1}{1+L_{i}(x)}$ is Optimal reference to LOGIT and CONVERGE TO REAL LIFE DEFAULT PROBABILITIES for each $1,2, \ldots, n$.

(ii) $\quad P D_{\text {Stressed }}=L_{i}(x)$ is Optimal reference to PROBIT and CONVERGE TO REAL LIFE DEFAULT PROBABILITIES for each $1,2, \ldots, n$.

(iii) Or the difference between the MODEL DEFAULTPROBABILITIES and REAL LIFEDEFAULT PROBABILITIES will be very NEGLIGIBLE or even possibly ZERO at many points in time $t$. Note that one can work out for the other proposed model TYPES.

\section{Empirical Results}

Assume, the data distribution Mean equal 0, Standard Deviation equal 1 for LOG-LOGISTIC (3P) $\equiv \boldsymbol{f}_{\mathbf{1}}(\boldsymbol{x})$, CAUCHY $\equiv \boldsymbol{f}_{\mathbf{2}}(\boldsymbol{x}) \quad$ and $\operatorname{BURR(4P)} \equiv \boldsymbol{f}_{\mathbf{3}}(\boldsymbol{x}) \quad$.While, $\mu_{A}=0.030383975 \quad, \quad$ and $\sigma_{A}=0.111414539$.Assume $\sum_{i=0}^{k} \beta_{i} X_{i}=\sum_{j=1}^{J} \beta_{j} X_{j}=0.4673$ and $\beta_{0}=0.0703$.

Then we canPredict Twenty One (21) working days (from 12/1/ 2014 to 12/30/ 2014) Default Probabilities andcompare it with NORMAL DEFAULT PROBABILITIES.

The Author performs the PREDICTION Using MICROSOFT EXCEL and obtained the following RESULTS as shown in Tables below:

Note that in Table 1: the notationT1-2D LOGIT ++ means Type 1 Two-Dimensional Logit(with respect to LogLogistic (3P) and Cauchy) ++ etc, Table 2: the notationT1-3D LOGIT +++ means Type 1 Three-Dimensional Logit(with respect to Log-Logistic (3P), Cauchy and Burr (4P)) +++ etc, Table 3: the notationT1-2D PROBIT ++ means Type 1 Two-Dimensional Probit(with respect to Log-Logistic (3P) and Cauchy) ++ etc and Table 4: the notationT1-3D PROBIT +++ means Type 1 Three-Dimensional Probit(with respect to Log-Logistic (3P), Cauchy and Burr (4P)) +++ etc.

Table 1. Propose Two-Dimensional Stressed Logit Default Probability Models TYPE 1

\begin{tabular}{|c|c|c|c|c|c|c|c|c|}
\hline Date & Time & Price & Return & Normal PD & T1-2D LOGIT ++ & T1-2D LOGIT -+ & T1-2D LOGIT+ - & T1-2D LOGIT- \\
\hline $12 / 1 / 2014$ & 1 & 111.730003 & 0.020288572 & 0.399106529 & 0.487854521 & 0.487874481 & 0.505333318 & 0.505354734 \\
\hline $12 / 2 / 2014$ & 2 & 114.019997 & -0.002722506 & 0.398945237 & 0.487860892 & 0.487861289 & 0.505347471 & 0.505347898 \\
\hline $12 / 3 / 2014$ & 3 & 113.709999 & -0.012655596 & 0.399006182 & 0.48785788 & 0.487866879 & 0.505341474 & 0.505351129 \\
\hline $12 / 4 / 2014$ & 4 & 112.279999 & -0.012637372 & 0.399005998 & 0.48785789 & 0.487866862 & 0.505341493 & 0.505351119 \\
\hline $12 / 5 / 2014$ & 5 & 110.870003 & -0.037400416 & 0.399500708 & 0.487828654 & 0.487916991 & 0.505287719 & 0.505382493 \\
\hline $12 / 8 / 2014$ & 6 & 106.800003 & 0.001964352 & 0.39894382 & 0.487860959 & 0.487861162 & 0.505347608 & 0.505347826 \\
\hline $12 / 9 / 2014$ & 7 & 107.010002 & -0.02029617 & 0.399106652 & 0.487852514 & 0.487876494 & 0.505331159 & 0.505356887 \\
\hline $12 / 10 / 2014$ & 8 & 104.860001 & 0.000476741 & 0.398942371 & 0.487861024 & 0.487861036 & 0.505347743 & 0.505347756 \\
\hline $12 / 11 / 2014$ & 9 & 104.910004 & -0.024411526 & 0.39918009 & 0.487848372 & 0.48788374 & 0.505323385 & 0.505361332 \\
\hline $12 / 12 / 2014$ & 10 & 102.379997 & -0.014957925 & 0.39903155 & 0.487856564 & 0.487869268 & 0.50533891 & 0.505352542 \\
\hline $12 / 15 / 2014$ & 11 & 100.860001 & 0.008293847 & 0.398969724 & 0.487859851 & 0.487863366 & 0.505345243 & 0.505349015 \\
\hline $12 / 16 / 2014$ & 12 & 101.699997 & 0.041600454 & 0.399633287 & 0.487837288 & 0.487913939 & 0.505290992 & 0.505373226 \\
\hline $12 / 17 / 2014$ & 13 & 106.019997 & 0.027995337 & 0.39925507 & 0.487849257 & 0.487886021 & 0.505320938 & 0.505360383 \\
\hline $12 / 18 / 2014$ & 14 & 109.029999 & 0.035145093 & 0.399435349 & 0.487843338 & 0.487899547 & 0.505306428 & 0.505366734 \\
\hline $12 / 19 / 2014$ & 15 & 112.93 & -0.008001474 & 0.398967823 & 0.487859808 & 0.487863329 & 0.505345283 & 0.505349061 \\
\hline $12 / 22 / 2014$ & 16 & 112.029999 & 0.016993046 & 0.399057497 & 0.487856363 & 0.487870567 & 0.505337517 & 0.505352758 \\
\hline $12 / 23 / 2014$ & 17 & 113.949997 & -0.004221236 & 0.398949389 & 0.487860697 & 0.48786166 & 0.505347074 & 0.505348107 \\
\hline $12 / 24 / 2014$ & 18 & 113.470001 & -0.001940729 & 0.398943783 & 0.487860959 & 0.48786116 & 0.50534761 & 0.505347826 \\
\hline $12 / 26 / 2014$ & 19 & 113.25 & 0.000617911 & 0.398942433 & 0.487861021 & 0.487861041 & 0.505347738 & 0.505347759 \\
\hline $12 / 29 / 2014$ & 20 & 113.32 & -0.00185487 & 0.398943653 & 0.487860965 & 0.487861149 & 0.505347622 & 0.505347819 \\
\hline
\end{tabular}


Table 2. Propose Three-Dimensional Stressed Logit Default Probability Models TYPE 1

\begin{tabular}{|c|c|c|c|c|c|c|c|c|}
\hline Normal PD & T1-3D LOGIT+++ & T1-3D LOGIT++- & T1-3D LOGIT- -+ & T1-3D LOGIT+-+ & T1-3D LOGIT-+- & T1-3D LOGIT--- & T1-3D LOGIT--+ & T1-3D LOGIT+-- \\
\hline 0.399106529 & 0.494713274 & 0.481183349 & 0.512718097 & 0.512696053 & 0.481202766 & 0.498199873 & 0.512718097 & 0.498179059 \\
\hline 0.398945237 & 0.494563064 & 0.481337942 & 0.512542693 & 0.512542254 & 0.48133833 & 0.4983523 & 0.512542693 & 0.498351885 \\
\hline 0.399006182 & 0.494492321 & 0.481399107 & 0.512473358 & 0.51246343 & 0.481407869 & 0.498424152 & 0.512473358 & 0.49841476 \\
\hline 0.399005998 & 0.494492455 & 0.481398999 & 0.512473481 & 0.512463582 & 0.481407735 & 0.498424016 & 0.512473481 & 0.498414652 \\
\hline 0.399500708 & 0.494294165 & 0.481530101 & 0.512324992 & 0.512227596 & 0.481616171 & 0.498625634 & 0.512324992 & 0.498533377 \\
\hline 0.39894382 & 0.494595066 & 0.481307764 & 0.512576915 & 0.512576691 & 0.481307962 & 0.498319811 & 0.512576915 & 0.498319599 \\
\hline 0.399106652 & 0.494434817 & 0.481443166 & 0.512423438 & 0.512396985 & 0.48146652 & 0.498482588 & 0.512423438 & 0.498457554 \\
\hline 0.398942371 & 0.494584996 & 0.481317426 & 0.512565957 & 0.512565944 & 0.481317438 & 0.498330032 & 0.512565957 & 0.49833002 \\
\hline 0.39918009 & 0.494402583 & 0.481465664 & 0.512397954 & 0.512358943 & 0.481500112 & 0.498515356 & 0.512397954 & 0.49847843 \\
\hline 0.39903155 & 0.494475297 & 0.481412679 & 0.512457979 & 0.512443962 & 0.48142505 & 0.498441449 & 0.512457979 & 0.498428188 \\
\hline 0.398969724 & 0.494637053 & 0.481265852 & 0.512624459 & 0.512620577 & 0.481269273 & 0.498277195 & 0.512624459 & 0.498273528 \\
\hline 0.399633287 & 0.494840408 & 0.481029622 & 0.512892748 & 0.512808048 & 0.481104149 & 0.498071006 & 0.512892748 & 0.497991131 \\
\hline 0.39925507 & 0.494760305 & 0.481128622 & 0.512780246 & 0.512739634 & 0.481164381 & 0.498152186 & 0.512780246 & 0.498113858 \\
\hline 0.399435349 & 0.494802815 & 0.481076918 & 0.51283899 & 0.512776888 & 0.481131579 & 0.498109098 & 0.51283899 & 0.498050511 \\
\hline 0.398967823 & 0.494525992 & 0.481370953 & 0.512505269 & 0.512501383 & 0.481374381 & 0.498389948 & 0.512505269 & 0.498386274 \\
\hline 0.399057497 & 0.494692727 & 0.481206371 & 0.51269196 & 0.512676274 & 0.481220191 & 0.498220712 & 0.51269196 & 0.498205898 \\
\hline 0.398949389 & 0.494552654 & 0.481347424 & 0.512531942 & 0.512530879 & 0.481348362 & 0.498362871 & 0.512531942 & 0.498361866 \\
\hline 0.398943783 & 0.494568459 & 0.481332963 & 0.512548339 & 0.512548117 & 0.481333159 & 0.498346822 & 0.512548339 & 0.498346612 \\
\hline 0.398942433 & 0.494585955 & 0.481316513 & 0.512566993 & 0.512566971 & 0.481316532 & 0.498329059 & 0.512566993 & 0.498329038 \\
\hline 0.398943653 & 0.49456905 & 0.481332415 & 0.512548961 & 0.512548758 & 0.481332594 & 0.498346222 & 0.512548961 & 0.49834603 \\
\hline
\end{tabular}

Table 3. Propose Two-Dimensional Stressed Probit Default Probability Models TYPE 1

\begin{tabular}{|c|c|c|c|c|c|c|c|c|}
\hline Date & Time & Price & Return & Normal PD & T1-2D LOGIT ++ & T1-2D LOGIT -+ & T1-2D LOGIT+- & T1-2D LOGIT-- \\
\hline $12 / 1 / 2014$ & 1 & 111.730003 & 0.020288572 & 0.399106529 & 0.542007891 & 0.541924029 & 0.471108377 & 0.471024515 \\
\hline $12 / 2 / 2014$ & 2 & 114.019997 & -0.002722506 & 0.398945237 & 0.541981125 & 0.541979453 & 0.471052953 & 0.471051281 \\
\hline $12 / 3 / 2014$ & 3 & 113.709999 & -0.012655596 & 0.399006182 & 0.541993776 & 0.54195597 & 0.471076436 & 0.47103863 \\
\hline $12 / 4 / 2014$ & 4 & 112.279999 & -0.012637372 & 0.399005998 & 0.541993737 & 0.541956042 & 0.471076364 & 0.471038669 \\
\hline $12 / 5 / 2014$ & 5 & 110.870003 & -0.037400416 & 0.399500708 & 0.542116582 & 0.541745447 & 0.471286959 & 0.470915824 \\
\hline $12 / 8 / 2014$ & 6 & 106.800003 & 0.001964352 & 0.39894382 & 0.541980841 & 0.541979989 & 0.471052417 & 0.471051564 \\
\hline $12 / 9 / 2014$ & 7 & 107.010002 & -0.02029617 & 0.399106652 & 0.542016324 & 0.541915574 & 0.471116832 & 0.471016082 \\
\hline $12 / 10 / 2014$ & 8 & 104.860001 & 0.000476741 & 0.398942371 & 0.541980569 & 0.541980519 & 0.471051887 & 0.471051837 \\
\hline $12 / 11 / 2014$ & 9 & 104.910004 & -0.024411526 & 0.39918009 & 0.542033729 & 0.541885133 & 0.471147273 & 0.470998677 \\
\hline $12 / 12 / 2014$ & 10 & 102.379997 & -0.014957925 & 0.39903155 & 0.541999309 & 0.54194593 & 0.471086476 & 0.471033097 \\
\hline $12 / 15 / 2014$ & 11 & 100.860001 & 0.008293847 & 0.398969724 & 0.541985498 & 0.541970728 & 0.471061678 & 0.471046908 \\
\hline $12 / 16 / 2014$ & 12 & 101.699997 & 0.041600454 & 0.399633287 & 0.5420803 & 0.541758268 & 0.471274138 & 0.470952106 \\
\hline $12 / 17 / 2014$ & 13 & 106.019997 & 0.027995337 & 0.39925507 & 0.542030011 & 0.541875547 & 0.471156859 & 0.471002395 \\
\hline $12 / 18 / 2014$ & 14 & 109.029999 & 0.035145093 & 0.399435349 & 0.542054879 & 0.541818724 & 0.471213682 & 0.470977527 \\
\hline $12 / 19 / 2014$ & 15 & 112.93 & -0.008001474 & 0.398967823 & 0.541985679 & 0.541970885 & 0.471061521 & 0.471046727 \\
\hline $12 / 22 / 2014$ & 16 & 112.029999 & 0.016993046 & 0.399057497 & 0.542000154 & 0.541940475 & 0.471091931 & 0.471032252 \\
\hline $12 / 23 / 2014$ & 17 & 113.949997 & -0.004221236 & 0.398949389 & 0.541981944 & 0.541977897 & 0.471054509 & 0.471050462 \\
\hline $12 / 24 / 2014$ & 18 & 113.470001 & -0.001940729 & 0.398943783 & 0.541980842 & 0.541979995 & 0.471052411 & 0.471051564 \\
\hline $12 / 26 / 2014$ & 19 & 113.25 & 0.000617911 & 0.398942433 & 0.541980581 & 0.541980496 & 0.47105191 & 0.471051825 \\
\hline $12 / 29 / 2014$ & 20 & 113.32 & -0.00185487 & 0.398943653 & 0.541980817 & 0.541980044 & 0.471052362 & 0.471051589 \\
\hline
\end{tabular}

Table 4. Propose Three-Dimensional Stressed Probit Default Probability Models TYPE 1

\begin{tabular}{|c|c|c|c|c|c|c|c|c|}
\hline Normal PD & T1-3D PROBIT+++ & T1-3D PROBIT++- & T1-3D PROBIT--+ & T1-3D PROBIT+-- & T1-3D PROBIT -+- & T1-3D PROBIT --- & T1-3D PROBIT --+ & T1-3D PROBIT +-- \\
\hline 0.399106529 & 0.513589384 & 0.577886715 & 0.442606008 & 0.44268987 & 0.570342536 & 0.499443021 & 0.442606008 & 0.499526883 \\
\hline 0.398945237 & 0.514203322 & 0.577887699 & 0.443273477 & 0.443275149 & 0.569757257 & 0.498829084 & 0.443273477 & 0.498830756 \\
\hline 0.399006182 & 0.51449259 & 0.577887328 & & 0.44357525 & 0.569457156 & 0.498539815 & 0.443537444 & 0.498577622 \\
\hline 0.399005998 & 0.514492043 & 0.577887329 & 0.443536976 & 0.443574671 & 0.569457735 & 0.498540362 & 0.443536976 & 0.498578057 \\
\hline 0.399500708 & 0.515303294 & 0.57788429 & 0.444102537 & 0.444473671 & 0.568558735 & 0.497729112 & 0.444102537 & 0.498100247 \\
\hline 0.39894382 & 0.514072493 & 0.577887708 & 0.443143216 & 0.443144068 & 0.569888338 & 0.498959913 & 0.443143216 & 0.498960766 \\
\hline
\end{tabular}




\begin{tabular}{|c|c|c|c|c|c|c|c|c|}
\hline 0.399106652 & 0.514727785 & 0.577886715 & 0.443727542 & 0.443828292 & 0.569204114 & 0.498304621 & 0.443727542 & 0.498405372 \\
\hline 0.398942371 & 0.514113656 & 0.577887716 & 0.443184923 & 0.443184973 & 0.569847433 & 0.49891875 & 0.443184923 & 0.498918801 \\
\hline 0.39918009 & 0.514859651 & 0.577886265 & 0.443824599 & 0.443973195 & 0.569059211 & 0.498172755 & 0.443824599 & 0.498321351 \\
\hline 0.39903155 & 0.514562215 & 0.577887173 & 0.443596004 & 0.443649382 & 0.569383023 & 0.498470191 & 0.443596004 & 0.49852357 \\
\hline 0.398969724 & 0.513900866 & 0.57788755 & 0.442962275 & 0.442977046 & 0.57005536 & 0.49913154 & 0.442962275 & 0.499146311 \\
\hline 0.399633287 & 0.513070053 & 0.577883468 & 0.44194186 & 0.442263892 & 0.570768514 & 0.499962353 & 0.44194186 & 0.500284385 \\
\hline 0.39925507 & 0.513397237 & 0.577885805 & 0.442369621 & 0.442524085 & 0.570508321 & 0.499635168 & 0.442369621 & 0.499789633 \\
\hline 0.399435349 & 0.513223589 & 0.577884694 & 0.442146237 & 0.442382392 & 0.570650014 & 0.499808817 & 0.442146237 & 0.500044972 \\
\hline 0.398967823 & 0.5143549 & 0.577887561 & 0.443415949 & 0.443430742 & 0.569601664 & 0.498677506 & 0.443415949 & 0.4986923 \\
\hline 0.399057497 & 0.513673341 & 0.577887015 & 0.442705439 & 0.442765118 & 0.570267288 & 0.499359065 & 0.442705439 & 0.499418745 \\
\hline 0.398949389 & 0.514245884 & 0.577887674 & 0.443314402 & 0.443318449 & 0.569713957 & 0.498786522 & 0.443314402 & 0.498790569 \\
\hline 0.398943783 & 0.514181263 & 0.577887708 & 0.443251985 & 0.443252832 & 0.569779574 & 0.498851143 & 0.443251985 & 0.498851989 \\
\hline 0.398942433 & 0.514109735 & 0.577887716 & 0.443180979 & 0.443181064 & 0.569851342 & 0.498922671 & 0.443180979 & 0.498922755 \\
\hline 0.398943653 & 0.514178847 & 0.577887709 & 0.443249619 & 0.443250392 & 0.569782014 & 0.498853559 & 0.443249619 & 0.498854332 \\
\hline
\end{tabular}

It can be observed, all the Shaded Areas in Table 1 to 4 shows NORMAL DEFAULT PROBABILITIES while the Un-shaded Areasshown the performances of the proposed Dimensional Default Probability Models vis-à-vis NORMAL DEFAULT PROBABILITIES. However, the scope of the research work is to compare the proposed Dimensional Default Probability Models with the Normal Default Probabilities as the Real LifeDefault Probabilities are NOT at the Author's disposal.

The results performances were FASCINATINGLY interesting, impressive, viable, reliable, sophisticated and complaint with IFRS 9 since they incorporated the forward-looking information satisfiesJameel's Criterion and Geometric average of only positive Economic forecasts of the future Macroeconomic scenarios $\left\{\mu_{A}>0\right.$, Inf initesimal and $\left.\left(\boldsymbol{\sigma}_{A} \geq \mathbf{1}\right)\right\}$ and also minimized the differences betweenModel Default Probabilities and REAL LIFE DEFAULT PROBABILITIES.

\section{Discussions}

In this paper, the performances of the PROPOSED MODELS with respect to LOG-LOGISTIC (3P), CAUCHY, and BURR (4P) can be improved using the following:

(1) Accurate prediction of economic forecasts of fundamental macroeconomic parameters used in the proposed models

(2) The Author set the Log-Logistic (3P) parameter $\xi$ to be 1 and Burr (4P) parameters $a=1, k=1, \gamma=$ $1, \beta=1$ and $\alpha=2$ thus collapsed to almost Normal. With HIGH VALUES of $\xi, a, k, \gamma, \beta$, and $\alpha$, the proposed Jameel's Stressed Closed Prices will effectively approximates the REAL PRICES.

(3) Jameel's Criterion axiom known as "Criterion Enhancement Axiom": That if we could be able to Runs the Goodness of Fit Tests such as the RANKS of Kolmogorov Smirnov (KS) Test, Anderson-Darling Test, Jarque and Bera (JB) Test, Shapiro Wilk (SW) Test, Cramer-Von Mises Test, Pearson $\left(\chi^{2}\right.$ Godness of Fit $)$ Test, Lilliefors Corrected K-S Test, D'AgostinoSkewness Test, Anscombe-Glynn Kurtosis Test, D'Agostino-Pearson Omnibus are all UNITY (1) of the underlying Stock Returns then the proposed Jameel's Stressed Closed Prices will coincide at finitely many points with the REAL PRICES .

(4) $\mu_{A}$ can be TESTED as ARITHMETIC Means of only positive Arithmetic Means of the Underlying Asset Return and Returns of the future economic forecasts of macroeconomic parameters, otherwise should remainsGEOMETRIC MEANS as defined and used in the paper.

\section{Conclusion}

LOGIT and PROBIT Models were first applied to Financial Markets byOhlson (1980) and Zmijewski (1984) to predict bankruptcy and to estimate probability of default respectively.Logit possesses FATTER TAILS than Probit and that makes it more robust to calculate Default probabilities. However, with the advancement in Information and Telecommunication Technology, Natural Disasters, Civil Unrest, Terrorism, Stock Market Crashes and Bubbles, Banks and other Institutions find it very difficult to accurately calculate Default Probabilities of their Borrowers, thus, Logit, Probit and other Default Probability Models needs to be enhanced to be abled accurately Quantify and Predict potential Credit Risk face by those Institutions.

Jamilu (2015) enhanced LOGIT and PROBIT Default Probability Models with the aid of one-dimensional forward-looking information $\{f(x)\}$ satisfies Jameel's Criterion and positive average of economic forecasts of 
future macroeconomic scenarios $\left\{\mu_{A}\right.$ and $\left.\sigma_{A}\right\}$. In this paper, the Author further enhance LOGIT and PROBIT Default Probability Models using Two and Three Dimensional Forward-Looking Information(s) $\left\{f_{1}(x), f_{2}(x)\right\}$ and $\left\{f_{1}(x), f_{2}(x), f_{3}(x)\right\}$ respectively satisfies Jameel's Criterion with LOG-LOGISTIC $(3 \mathrm{P}) \equiv f_{1}(x)$, CAUCHY $\equiv f_{2}(x)$ and $\operatorname{BURR}(4 \mathrm{P}) \equiv f_{3}(x)$ and positive average of economic forecasts of future macroeconomic scenarios $\left\{\mu_{A}\right.$ and $\left.\sigma_{A}\right\}$. The paper tested the performances of only proposed Default Probability Models of TYPE 1 in each class using Twenty One (21) working days (from 12/1/ 2014 to 12/30/ 2014). The results were fascinatingly interesting, impressive, viable and reliable, sophisticated, and complaint with IFRS 9 since they incorporated forward-looking information and Economic forecasts of the future macroeconomic scenarios thereby minimizing the differences between MODELS DEFAULT PROBABILITIES and REAL LIFE DEFAULT PROBABILITIES.

Also, Jameel's n-dimensional Stressed Default Probability Theorem was proposed in the paper, however, the Theorem is expected to sufficiently approximate REAL LIFE Default Probabilities.

In the case of future research direction, one can use the Theorem to find ARBITRARY COMBINATIONS EXCLUDING FIRST FEW TERMS OF DIFFERENT DIMENSIONS $L_{j}(x)$ among $\left\{L_{1}(x), L_{2}(x)\right.$, $\left.L_{3}(x), \ldots, L_{n}(x)\right\}$ such that $L_{j}(x)-P_{i}(x)=0$ at every point $, j=1,2, \ldots, m, i=1,2, \ldots, k, 0 \leq P_{i}(x) \leq$ 1 where $P_{i}(x), x$ is the Asset's Return is the REAL LIFE Default Probabilities correspond to eachMODEL Default Probabilities $L_{i}(x)$.

\section{Reference}

Adegoke, O. (1979). Introduction to Real Analysis, Department of Mathematics, University of Ibadan, Girardet Press (W.A) Co; Ibadan.

Andrew, W. L., \& Graig, M. A. (1999). A Non-Random Walk Down Wall Street, Princeton University Press, 41 William Street Princeton, New Jersey 08540, United Kingdom.

Arditti, F. D. (1996). Derivatives: A Comprehensive Resource for Options, Futures, Interest Rate Swaps, and Mortgage Securities. Boston: Harvard Business School Press. ISBN0-87584560-6.

Barnaby, B., et al. (2016). Complying with IFRS 9 Impairment Calculations for Retail Portfolios, Moody's Analytics Risk Perspectives, the convergence of Risk, Finance, and Accounting, Volume VII, June, 2016.

Bellotti, T., \& Crook, J. (2012). Loss Given Default Models Incorporating Macroeconomic Variables for Credit Cards. International Journal of Forecasting, 28(1), 171-182. https://doi.org/10.1016/j.ijforecast.2010.08.005

Bhatt, B. J., et al (2013). Fractional Brownian Motion and Predictability Index in Financial Market, Global Journal of Mathematical Sciences: Theory and Practical, Volume 5, Number 3 (2013), pp. 197-203.

Borio, C., \& Lowe, P. (2001). To Provision or not to Provision. BIS Quarterly Review, 9(3), 36-48.

Burton, G. M. (1999). A Random Walk Down Wall Street, W.W. Norton \& Company, Inc. 500 fifth Avenue, New York, N. Y. 10110.

Burton, G. M. (2009). The Clustering of Extreme Movements: Stock prices and the Weather, Princeton University, AtanuSaha, Alixpartners, Alex Grecu, Huron Consulting Group, CEPS working paper No. 186 February, 2009.

Daniel, P. (2006). Estimating Probabilities of Default for German Savings Banks and Credit Cooperatives, University of Applied Sciences, Mainz, Ander Bruchspitze 50, D - 55122 Mainz.

David, M. R. (2012). Simulating Default Probabilities in Stress Scenarios, Presented to the PRMIA Global Risk Conference, New York, NY, May 14, 2012.

David, N. (2006). Fractional Brownian Motion: Stochastic Calculus and Applications; Proceedings of the International Congress of Mathematicians, Madrid, Spain, 2006, European Mathematical Society

EwaBialkowska \& Chai, A. (2017), Banks-IFRS 9 Pre-transition Disclosure: Guide to Annual Financial Statements for Banks, KPMG, IFRG Limited, 1-18.

Jamilu A. A. (2013). A Guide to Financial Mathematics and Risk Management for Nigeria, Book Published by Delcon Press, Suleja, Niger, ISBN: 978 - 223 - 529 - 6, First Edition 2013.

Jamilu A. A. (2014). Modern Approach to Financial Risk Management, Book Published by Delcon Press, Suleja, Niger, ISBN: 978-978-942-265-4, First Edition 2014.

Jamilu, A. A. (2014). Understanding Financial Risks, Book Published by Delcon Press, Suleja, Niger, ISBN: ISBN: 978-978-942-266-1, First Edition 2014.

Jamilu, A. A. (2015). Banking and Economic Advanced Stressed Probability of Default Models, Accepted for 
Publication in the Asian Journal of Management Sciences, AJMS/2015/ May - June - $303-798-2$ - RV.

Jamilu, A. A. (2018). IFRS 9 Measurement of Financial Instruments 2018: Jameel's Non-Normal Brownian Motion Models are Indeed IFRS 9 Complaint Models. Journal of Economics and Management Sciences, 1(1), 92-113. https://doi.org/10.30560/jems.v1n1p92

Jamilu, A. A. (2018). Jameel's Two and Three-Dimensional Stressed Closed Form Models are Indeed IFRS 9 Complaint Models. Journal of Economics and Management Sciences, 1(1), $175-191$. https://doi.org/10.30560/jems.v1n1p175

Jamilu, A. A.(2015). Estimation of Probability of Default using Advanced Stressed Probability of Default Models, Ongoing Ph.D Thesis, Ahmadu Bello University (ABU), Zaria, Nigeria.

Krishna, R., \& Vaughan, C. (2016). Simulating Stock Prices using Geometric Brownian Motion: Evidence from Australian Companies, Australasian Accounting. Business and Finance Journal, 10(3) 2016, 23-47. https://doi.org/10.14453/aabfj.v10i3.3

Nassim, N. T. (2007). Black Swans and the Domains of Statistics, American Statistician, August 2007, 6I(3).

Nassim, N. T. (2009). Errors, Robustness, and Fourth Quadrant, New York University Polytechnic Institute and Universa Investment, United States. International Journal of Forecasting, 25(2009), 744-759. https://doi.org/10.1016/j.ijforecast.2009.05.027

Nassim, N. T. (2010). Convexity, Robustness, and Model Error inside the "Black Swan Domain", Draft Version, September, 2010.

Nassim, N. T. (2010). Why Did the Crisis of 2008 Happen, Draft, $3^{\text {rd }}$ Version, August, 2010.

Nassim, N. T. (2011), The Future has Thicker Tails Past: Model Error as Branching Counterfactuals, presented in Honor of Benoit Mandelbrot's at his Scientific Memorial, Yale University, April, 2011.

Nassim, N. T. (2012). The Illusion of Thin - Tails under Aggregation, NYU - Poly, January, 2012.

Nassim, N. T.(2011). A Map and Simple Heuristic to Detect Fragility, Antifragility, and Model Error, First Version, 2011.

Nassim, N. T., et al (2009). Risk Externalities and Too bid to Fail, New York University Polytechnic Institute, 11201, New York, United States.

Onali, E., \& Ginesti, G. (2014). Pre-adoption Market Reaction to IFRS 9: A Cross-country Event-study. Journal of Accounting and Public Policy, 33(6), 628-637. https://doi.org/10.1016/j.jaccpubpol.2014.08.004

Reney, D. E., \& Michael, R. M. (2016). Forecasting of Stock Prices using Brownian Motion - Monte Carlo Simulation, Proceedings of the 2016 International Conference on Industrial Engineering and Operations Management, Kuala Lumpur, Malaysia, March 8-10, 2016.

Rogers, L. C. G. (1997). Arbitrage with Fractional Brownian Motion, Mathematical Finance, Vol.7, No.1 (January, 1997), 95-105. https://doi.org/10.1111/1467-9965.00025

Spreedhar T Bharath et al (2004). Forecasting Default with the KMV - Merton Model, University of Michigan, Ann Arbor MI 48109.

Steven, R. D. (2016). Stochastic Processes and Advanced Mathematical Finance, The Definition of Brownian Motion and the Wiener process, Department of Mathematics, 203 Avery Hall, University of Nebraska-Lincoln, Lincoln, NE 68588-0130.

Tidaruk, A,(2014). Mathematical Model of Stock Prices via a Fractional Brownian Motion Model with Adaptive Parameters.

Ton, D. (2004). Simulation of Fractional Brownian Motion, Thesis, University of Twente, Department of Mathematical Sciences, P.O. BOX 217, 7500 AE Enschede, Netherlands.

Van Ness, M. (1968). Fractional Brownian Motions, Fractional Noises and Applications (M \& Van Ness (1968)), SIAM Review: 10, 1968, 422-437. https://doi.org/10.1137/1010093

Wang, B., et al. (2012). When Brownian Diffusion is no Gaussian, Nature Materials, VOL 11, June, 2012 , Macmillian Publishers Limited.

Zhang, W. Y. (2015). Introduction to Ito's Lemma, Lecture Note, Cornell University, Department of Statistical Sciences, May 6, 2015. 


\section{Copyrights}

Copyright for this article is retained by the author(s), with first publication rights granted to the journal.

This is an open-access article distributed under the terms and conditions of the Creative Commons Attribution license (http://creativecommons.org/licenses/by/4.0/). 